M. Iosifescu · C. Kraaikamp

\title{
A three-dimensional probability distribution in the metrical theory of continued fractions
}

Received August 15, 2003

Abstract. Let $a_{n}, n \geq 1$, denote the incomplete quotients of the continued fraction expansion of an arbitrary irrational number in the unit interval $I=[0,1]$. For any $a \in I$ put $s_{n+1}^{a}=1 /\left(a_{n+1}+s_{n}^{a}\right)$, $u_{n+1}^{a}=s_{n}^{a}+1 / \tau^{n}, n \geq 0$, with $s_{0}^{a}=a$, where $\tau$ is the continued fraction transformation, and let $\gamma_{a}$ be the probability measure on the Borel subsets of $I$ defined by its distribution function

$$
\gamma_{a}([0, x])=\frac{(a+1) x}{a x+1}, \quad x \in I .
$$

We study the joint distribution function of $s_{n}^{a}, \tau^{n}$, and $u_{n+1}^{a}, n \geq 0$, under $\gamma_{a}, a \in I$. We derive the asymptotic distribution function, lower and upper bounds for the error as well as its optimal convergence rate to 0 as $n \rightarrow \infty$. The same problems are taken up for the distributions of the pairs $\left(\tau^{n}, u_{n+1}^{a}\right)$ and $\left(s_{n}^{a}, u_{n+1}^{a}\right)$ under $\gamma_{a}, a \in I$.

\section{Introduction and statement of the problem}

Let $\Omega \subset I=[0,1]$ be the set of irrationals in the unit interval. Consider the continued fraction transformation $\tau: \Omega \rightarrow \Omega$ defined by $\tau(\omega)=1 / \omega \bmod 1$ (that is, the fractionary part of $1 / \omega)$. Put $\mathbb{N}_{+}=\{1,2, \ldots\}$ and $\mathbb{N}=\mathbb{N}_{+} \cup\{0\}$. Define $a_{n+1}(\omega)=$ $a_{1}\left(\tau^{n}(\omega)\right), n \in \mathbb{N}, \omega \in \Omega$, where $\tau^{n}$ denotes the composition of $\tau$ with itself $n \in \mathbb{N}_{+}$ times, $\tau^{0}=$ identity map, and $a_{1}(\omega)=$ integer part of $1 / \omega, \omega \in \Omega$. Then

$$
\omega=\frac{\frac{1}{a_{1}(\omega)+\tau(\omega)}=\ldots}{a_{1}(\omega)+\frac{1}{a_{2}(\omega)+\ddots+\frac{1}{a_{n}(\omega)+\tau^{n}(\omega)}}}, \quad n \geq 2,
$$

The first author was supported by the Nederlandse Organisatie voor Wetenschappelijk Onderzoek (NWO).

M. Iosifescu: Romanian Academy, "Gheorghe Mihoc-Caius Iacob" Institute of Mathematical Statistics and Applied Mathematics, Casa Academiei Romane, Calea 13 Septembrie nr. 13, RO-050711 Bucharest 5, Romania; e-mail: miosifes@ valhalla.racai.ro

C. Kraaikamp: Technische Universiteit Delft, EWI (CROSS), Thomas Stieltjes Institute for Mathematics, Mekelweg 4, 2628 CD Delft, The Netherlands; e-mail: c.kraaikamp@its.tudelft.nl 
and we have

$$
\omega=\lim _{n \rightarrow \infty} \frac{p_{n}(\omega)}{q_{n}(\omega)}:=\left[a_{1}(\omega), a_{2}(\omega), \ldots\right], \quad \omega \in \Omega
$$

where

$$
\frac{p_{n}(\omega)}{q_{n}(\omega)}=\frac{1}{a_{1}(\omega)+\frac{1}{a_{2}(\omega)+\cdot+\frac{1}{a_{n}(\omega)}}}
$$

with g.c.d. $\left(p_{n}(\omega), q_{n}(\omega)\right)=1, \omega \in \Omega, n \in \mathbb{N}_{+}$.

The $a_{n}, n \in \mathbb{N}_{+}$, are clearly $\mathbb{N}_{+}$-valued random variables on $\left(I, \mathcal{B}_{I}\right)$, where $\mathcal{B}_{I}$ is the collection of Borel subsets of $I$, which are defined almost surely with respect to any probability measure on $\mathcal{B}_{I}$ assigning measure 0 to the set $I \backslash \Omega$ of rationals in $I$. An example of such a probability measure is Lebesgue measure $\lambda$, but a more important one in our context is the Gauss measure $\gamma$ defined by

$$
\gamma(A)=\frac{1}{\log 2} \int_{A} \frac{d x}{x+1}, \quad A \in \mathcal{B}_{I} .
$$

We have $\gamma=\gamma \tau^{-1}$, that is, $\gamma(A)=\gamma\left(\tau^{-1}(A)\right), A \in \mathcal{B}_{I}$. Therefore, by its very definition, the sequence $\left(a_{n}\right)_{n \in \mathbb{N}_{+}}$is strictly stationary on $\left(I, \mathcal{B}_{I}, \gamma\right)$.

There is also a whole family $\left(\gamma_{a}\right)_{a \in I}$ of probability measures on $\mathcal{B}_{I}$ defined by their distribution functions

$$
\gamma_{a}([0, x])=\frac{(a+1) x}{a x+1}, \quad x \in I, a \in I,
$$

which plays an important part. In particular, $\gamma_{0}=\lambda$. For any $a \in I$ and $n \in \mathbb{N}_{+}$we have

$$
\gamma_{a}\left(\tau^{n}<x \mid a_{1}, \ldots, a_{n}\right)=\frac{\left(s_{n}^{a}+1\right) x}{s_{n}^{a} x+1}, \quad x \in I,
$$

(see Proposition 1.3.8 in [IK]), where the $s_{n}^{a}$ are defined recursively by $s_{0}^{a}=a$ and

$$
s_{n+1}^{a}=\frac{1}{a_{n+1}+s_{n}^{a}}, \quad a \in I, n \in \mathbb{N} .
$$

Noting that $\tau^{n}(\omega)=\left[a_{n+1}(\omega), a_{n+2}(\omega), \ldots\right], n \in \mathbb{N}, \omega \in \Omega$, it follows that

$$
\gamma_{a}\left(a_{n+1}=i \mid a_{1}, \ldots, a_{n}\right)=\frac{s_{n}^{a}+1}{\left(s_{n}^{a}+i\right)\left(s_{n}^{a}+i+1\right)}=: P_{i}\left(s_{n}^{a}\right)
$$

for any $a \in I$ and $i, n \in \mathbb{N}_{+}$. Hence for any $a \in I$ the sequence $\left(s_{n}^{a}\right)_{n \in \mathbb{N}}$ on $\left(I, \mathcal{B}_{I}, \gamma_{a}\right)$ is an $I$-valued Markov chain which starts at $s_{0}^{a}=a$ and has the following transition mechanism: from state $s \in I$ the possible transitions are to any state $1 /(s+i)$ with corresponding transition probability $P_{i}(s), i \in \mathbb{N}_{+}$.

Finally, let us define the $[1, \infty)$-valued random variables

$$
u_{n+1}^{a}=s_{n}^{a}+\frac{1}{\tau^{n}}, \quad n \in \mathbb{N}, a \in I .
$$


In particular, we can write

$$
u_{n+1}^{0}(\omega)=q_{n}^{-2}\left|\omega-\frac{p_{n}(\omega)}{q_{n}(\omega)}\right|^{-1}, \quad \omega \in \Omega, n \in \mathbb{N}
$$

with $p_{0}(\omega)=0, q_{0}(\omega)=1, \omega \in \Omega$ (cf. [IK, p. 15]). Therefore, the quantities $\Theta_{n}:=$ $1 / u_{n+1}^{0}, n \in \mathbb{N}$, yield some information about the quality of the approximations $p_{n}(\omega) / q_{n}(\omega), n \in \mathbb{N}$, for $\omega$. Consider the distribution function $H$ on $[1, \infty)$ defined by

$$
H(z)= \begin{cases}\frac{1}{\log 2}\left(\log z-\frac{z-1}{z}\right) & \text { if } 1 \leq z \leq 2, \\ \frac{1}{\log 2}\left(\log z-\frac{1}{z}\right) & \text { if } z \geq 2\end{cases}
$$

The following results hold (cf. [IK] pp. 117-119, 154, and 98]).

(i) For any $a, x \in I$ and $n \in \mathbb{N}_{+}$we have

$$
\left|\gamma_{a}\left(\tau^{n}<x\right)-\frac{\log (x+1)}{\log 2}\right| \leq\left(\frac{\pi^{2} \log 2}{6}-1\right) \lambda_{0}^{n-1}\left(\frac{1}{2}-\left|\frac{1}{2}-\frac{\log (x+1)}{\log 2}\right|\right),
$$

where $\lambda_{0}=0.303663007898 \ldots$ (Wirsing's constant). For any $a \in I, a \neq a_{0}$, with $a_{0}$ very close to 0.4 , the exact convergence rate to 0 as $n \rightarrow \infty$ of

$$
\sup _{x \in I}\left|\gamma_{a}\left(\tau^{n}<x\right)-\frac{\log (x+1)}{\log 2}\right|
$$

is $\mathcal{O}\left(\lambda_{0}^{n}\right)$. For $a=a_{0}$ the rate is $\mathcal{O}\left(\lambda_{3}^{n}\right)$, with $\lambda_{3}=0.100884509293 \ldots$. Note that these results needed pretty nearly 162 years to be reached! See [IK, Ch. 2].

(ii) For any $a \in I,(x, y) \in I^{2}$, and $n \in \mathbb{N}$ we have

$$
\begin{aligned}
\frac{a+1}{2\left(\mathrm{~F}_{n}+a \mathrm{~F}_{n-1}\right)\left(\mathrm{F}_{n+1}+a \mathrm{~F}_{n}\right)} & \leq \sup _{x, y \in I}\left|\gamma_{a}\left(s_{n}^{a} \leq x, \tau^{n} \leq y\right)-\frac{\log (x y+1)}{\log 2}\right| \\
& \leq \frac{\kappa_{0}}{\mathrm{~F}_{n} \mathrm{~F}_{n+1}} .
\end{aligned}
$$

Here the $\mathrm{F}_{n}, n \in \mathbb{N}$, are the Fibonacci numbers defined recursively by $\mathrm{F}_{-1}=0, \mathrm{~F}_{0}=1$, $\mathrm{F}_{n+1}=\mathrm{F}_{n}+\mathrm{F}_{n-1}, n \in \mathbb{N}$, and $\kappa_{0}$ is a constant not exceeding 14.8. Hence, setting $G_{n}^{a}(x)=\gamma_{a}\left(s_{n}^{a} \leq x\right), a, x \in I, n \in \mathbb{N}$, and letting $y=1$, we obtain the same lower and upper bounds as above for

$$
\sup _{x \in I}\left|G_{n}^{a}(x)-\frac{\log (x+1)}{\log 2}\right| \text {. }
$$

These facts imply that for any $a \in I$ the exact convergence rates to 0 as $n \rightarrow \infty$ of both

and

$$
\sup _{x, y \in I}\left|\gamma_{a}\left(s_{n}^{a} \leq x, \tau^{n} \leq y\right)-\frac{\log (x y+1)}{\log 2}\right|
$$

$$
\sup _{x \in I}\left|G_{n}^{a}(x)-\frac{\log (x+1)}{\log 2}\right|
$$

are $\mathcal{O}\left(g^{2 n}\right)$, with $g=\frac{\sqrt{5}-1}{2}, g^{2}=\frac{3-\sqrt{5}}{2}=0.38196 \ldots$ 
(iii) For any $z \geq 1$ and $n \in \mathbb{N}$ we have

$$
\sup _{a \in I}\left|\gamma_{a}\left(u_{n+1}^{a} \leq z\right)-H(z)\right| \leq \frac{\kappa_{1} I_{(1, \infty)}(z)}{z} \lambda_{0}^{n},
$$

where $\kappa_{1}$ is a constant not exceeding 6.5 and $I_{(1, \infty)}$ stands for the indicator function of the infinite interval $(1, \infty)$.

The exact convergence rate to 0 as $n \rightarrow \infty$ of

$$
\sup _{z \in I}\left|\gamma_{a}\left(u_{n+1}^{a} \leq z\right)-H(z)\right|
$$

is $\mathcal{O}\left(\lambda_{0}^{n}\right)$ for any $a \in I \backslash E$, with $E=\left\{a \in I: \int_{I} \gamma(d x) \int_{a}^{x} \psi(y) d y=0\right\}$, where $\psi$ is a certain real-valued continuous function on $I$. See [IK, pp. 91 and 99]. For $a \in E$ the convergence rate to 0 as $n \rightarrow \infty$ of the above quantity is $\mathcal{O}\left(\alpha^{n}\right)$ for some $\alpha<\lambda_{0}$. Neither 0 nor 1 belongs to $E$.

In this paper we study the joint distribution of $s_{n}^{a}, \tau^{n}$, and $u_{n+1}^{a}, n \in \mathbb{N}$, under $\gamma_{a}$, $a \in I$. Even if there is a deterministic relationship connecting these variables, the picture emerging is an interesting one. We derive the asymptotic distribution function, lower and upper bounds for the error as well as its optimal convergence rate to 0 as $n \rightarrow \infty$, which is $\mathcal{O}\left(g^{2 n}\right)$. The same optimal convergence rate as $n \rightarrow \infty$ also holds for the marginal joint distribution under $\gamma_{a}$ of $s_{n}^{a}$ and $u_{n+1}^{a}$. Instead, and this is a notable exception, the optimal convergence rate as $n \rightarrow \infty$ for the marginal joint distribution under $\gamma_{a}$ of $\tau^{n}$ and $u_{n+1}^{a}$ is $\mathcal{O}\left(\lambda_{0}^{n}\right)$ for any $a \in I \backslash E$.

\section{Main result}

On $I^{2} \times[1, \infty)$ consider the distribution function $L$ defined by

$$
L(x, y, z)= \begin{cases}\frac{1}{\log 2}\left(\log (x y+1)-\frac{x}{z}\right) & \text { if } y \geq 1 /(z-x), \\ \frac{1}{\log 2}\left(\log y z+\frac{1}{y z}-1\right) & \text { if } 1 / z \leq y \leq 1 /(z-x), \\ 0 & \text { if } y \leq 1 / z\end{cases}
$$

for $(x, y) \in I^{2}$ and $z \geq 1$. Put

$$
H_{n}^{a}(x)=G_{n}^{a}(x)-\frac{\log (x+1)}{\log 2}, \quad a, x \in I, n \in \mathbb{N} .
$$

We can now state our main result as follows.

Theorem 1. For any $a \in I,(x, y) \in I^{2}$, and $z \geq 1$ the quantity

$$
\left|\gamma_{a}\left(s_{n}^{a} \leq x, \tau^{n} \leq y, u_{n+1}^{a} \leq z\right)-L(x, y, z)\right|
$$

is dominated by

(i) $\left(y-\frac{1}{z}\right) \sup _{0 \leq u \leq x}\left|H_{n}^{a}(u)\right| \quad$ if $y \geq 1 /(z-x)$ and $z\left(y-y^{2}\right) \leq 1$; 
(ii) $\left(\frac{2}{y z}\left(\sqrt{z\left(u-y^{2}\right)}-1\right)^{2}+y-\frac{1}{z}\right) \sup _{0 \leq u \leq x}\left|H_{n}^{a}(u)\right|$

$$
\text { if } y \geq 1 /(z-x) \text { and } 1 \leq z\left(y-y^{2}\right) \leq(x y+1)^{2} \text {; }
$$

(iii) $\left(2(x+1)\left(\frac{y}{x y+1}-\frac{1}{z}\right)-y+\frac{1}{z}\right) \sup _{0 \leq u \leq x}\left|H_{n}^{a}(u)\right|$

$$
\text { if } y \geq 1 /(z-x) \text { and } z\left(y-y^{2}\right)>(x y+1)^{2} \text {; }
$$

(iv) $\left(y-\frac{1}{z}\right) \sup _{0 \leq u \leq z-1 / y}\left|H_{n}^{a}(u)\right| \quad$ if $1 / z \leq y \leq 1 /(z-x)$ and $z\left(y-y^{2}\right) \leq 1$;

(v) $\left(\frac{2}{y z}\left(\sqrt{z\left(y-y^{2}\right)}-1\right)^{2}+y-\frac{1}{z}\right) \sup _{0 \leq u \leq z-1 / y}\left|H_{n}^{a}(u)\right|$

$$
\text { if } 1 / z \leq y \leq 1 /(z-x) \text { and } z\left(y-y^{2}\right) \geq 1 \text {; }
$$

(vi) $0 \quad$ if $y \leq 1 / z$.

Consequently, for every $a \in I$ the quantity

$$
\sup _{x, y \in I, z \geq 1}\left|\gamma_{a}\left(s_{n}^{a} \leq x, \tau^{n} \leq y, u_{n+1}^{a} \leq z\right)-L(x, y, z)\right|
$$

is dominated by $2 \kappa_{0} / \mathrm{F}_{n} \mathrm{~F}_{n+1} \leq 29.6 / \mathrm{F}_{n} \mathrm{~F}_{n+1}, n \in \mathbb{N}$, and is thus $\mathcal{O}\left(g^{2 n}\right)$ as $n \rightarrow \infty$. For any $a \in I$ and $n \in \mathbb{N}$ it exceeds

$$
\frac{a+1}{2\left(\mathrm{~F}_{n}+a \mathrm{~F}_{n-1}\right)\left(\mathrm{F}_{n+1}+a \mathrm{~F}_{n}\right)},
$$

which is also $\mathcal{O}\left(g^{2 n}\right)$ as $n \rightarrow \infty$. Therefore, the exact convergence rate to 0 of (4) as $n \rightarrow \infty$ is $\mathcal{O}\left(g^{2 n}\right)$.

Proof. Write

$$
\gamma_{a}\left(s_{n}^{a} \leq x, \tau^{n} \leq y, u_{n+1}^{a} \leq z\right)=P_{n}^{a}(x, y, z)
$$

and

$$
A(x, y, z)=\left\{(u, v) \in I^{2}: 0 \leq u \leq x, 0 \leq v \leq y, u+\frac{1}{v} \leq z\right\}
$$

for $a, x, y \in I, z \geq 1$, and $n \in \mathbb{N}$. By (2) we clearly have

$$
P_{n}^{a}(x, y, z)=\iint_{[0, x] \times[0, y]} I_{[1, z]}\left(u+\frac{1}{v}\right) d G_{n}^{a}(u, v),
$$

where $I_{[1, z]}$ stands for the indicator function of the segment $[1, z]$ and $G_{n}^{a}(u, v)=$ $P_{n}^{a}(u, v, \infty)$. Since by $(1)$ we have

$$
d G_{n}^{a}(u, v)=\frac{(u+1) d v}{(u v+1)^{2}} d G_{n}^{a}(u), \quad u, v \in I,
$$

it follows that

$$
P_{n}^{a}(x, y, z)=I_{1}+I_{2}
$$


where

$$
I_{1}=\frac{1}{\log 2} \iint_{A(x, y, z)} \frac{d u d v}{(u v+1)^{2}}, \quad I_{2}=\iint_{A(x, y, z)} \frac{(u+1) d v}{(u v+1)^{2}} d H_{n}^{a}(u) .
$$

It is clear that $A(x, y, z)=\emptyset$ if $y \leq 1 / z$. We shall then consider two cases (cf. Figures 1 and 2):

I $\quad y \geq 1 /(z-x),(x, y) \in I^{2}, z \geq 1$;

II $\quad 1 / z \leq y \leq 1 /(z-x),(x, y) \in I^{2}, z \geq 1$.
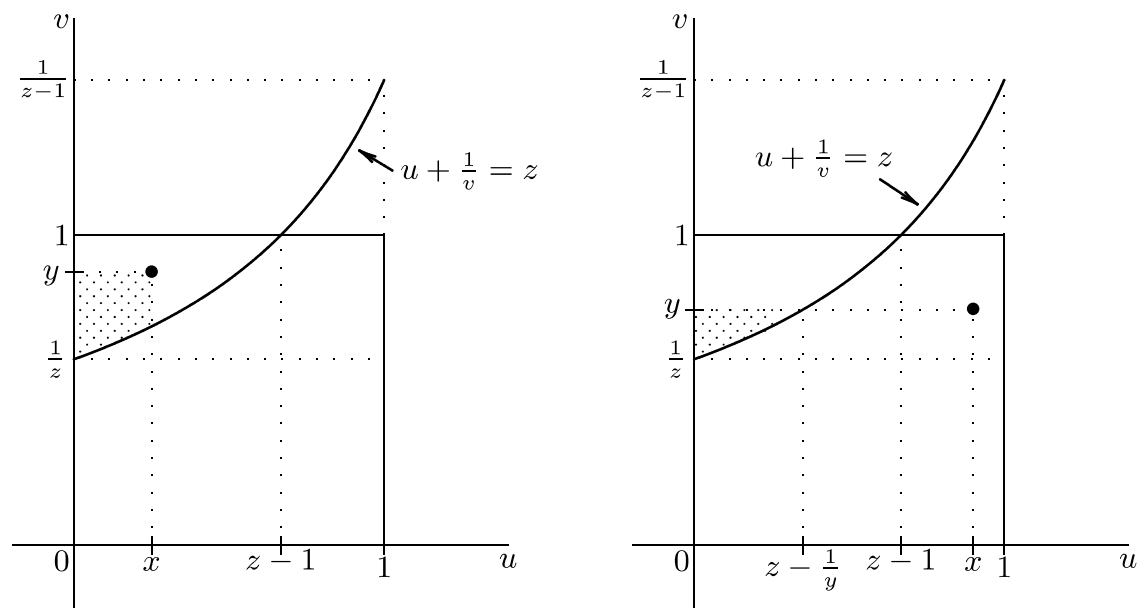

Fig. 1. $A(x, y, z)$ for $1<z<2$. Left: Case I; Right: Case II. (Here $z=5 / 3$.)
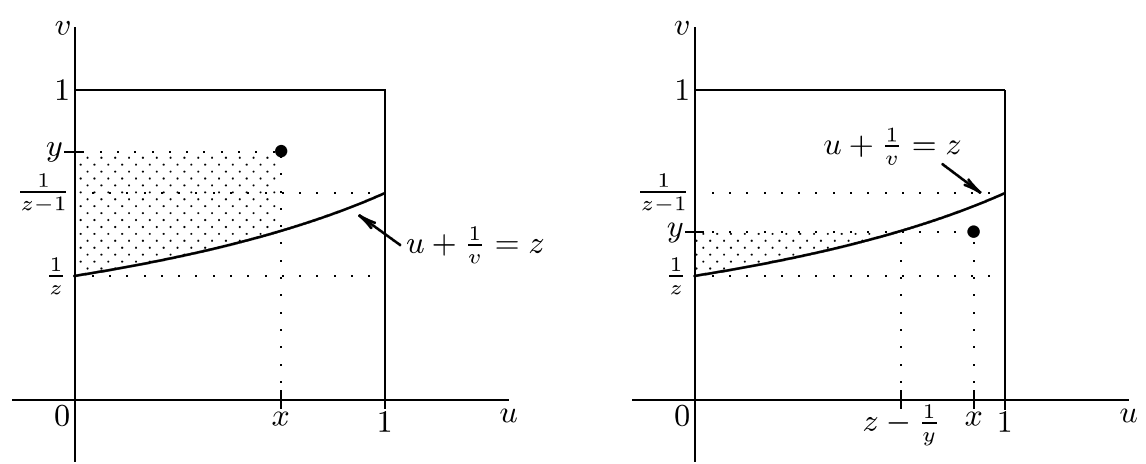

Fig. 2. $A(x, y, z)$ for $z>2$. Left: Case I; Right: Case II. (Here $z=5 / 2$.)

In Case I we have

$$
I_{1}=\frac{1}{\log 2} \int_{0}^{x} d u \int_{1 /(z-u)}^{y} \frac{d v}{(u v+1)^{2}}=\frac{1}{\log 2}\left(\log (x y+1)-\frac{x}{z}\right)
$$


while in Case II

$$
I_{1}=\frac{1}{\log 2} \int_{0}^{z-1 / y} d u \int_{1 /(z-u)}^{y} \frac{d v}{(u v+1)^{2}}=\frac{1}{\log 2}\left(\log y z+\frac{1}{y z}-1\right) .
$$

Next, in Case I we have

$$
\begin{aligned}
I_{2} & =\int_{0}^{x}(u+1) d H_{n}^{a}(u) \int_{1 /(z-u)}^{y} \frac{d v}{(u v+1)^{2}} \\
& =\int_{0}^{x}(u+1)\left(\frac{y}{u y+1}-\frac{1}{z}\right) d H_{n}^{a}(u) \\
& =\left.(u+1)\left(\frac{y}{u y+1}-\frac{1}{z}\right) H_{n}^{a}(u)\right|_{u=0} ^{u=x}-\int_{0}^{x} H_{n}(u)\left(\frac{y-y^{2}}{(u y+1)^{2}}-\frac{1}{z}\right) d u \\
& =(x+1)\left(\frac{y}{x y+1}-\frac{1}{z}\right) H_{n}^{a}(x)-\int_{0}^{x} H_{n}(u)\left(\frac{y-y^{2}}{(u y+1)^{2}}-\frac{1}{z}\right) d u .
\end{aligned}
$$

Putting

$$
u_{0}=\frac{\sqrt{z\left(y-y^{2}\right)}-1}{y}
$$

we then have

$$
\begin{aligned}
& \int_{0}^{x}\left|\frac{y-y^{2}}{(u y+1)^{2}}-\frac{1}{z}\right| d u \\
& = \begin{cases}\int_{0}^{x}\left(-\frac{y-y^{2}}{(u y+1)^{2}}+\frac{1}{z}\right) d u & \text { if } z\left(y-y^{2}\right) \leq 1, \\
\int_{0}^{u_{0}}\left(\frac{y-y^{2}}{(u y+1)^{2}}-\frac{1}{z}\right) d u & +\int_{u_{0}}^{x}\left(\frac{1}{z}-\frac{y-y^{2}}{(u y+1)^{2}}\right) d u \\
\int_{0}^{x}\left(\frac{y-y^{2}}{(u y+1)^{2}}-\frac{1}{z}\right) d u & \text { if } 1 \leq z\left(y-y^{2}\right) \leq(x y+1)^{2}, \\
& = \begin{cases}y-\frac{1}{z}-(x+1)\left(\frac{y}{x y+1}-\frac{1}{z}\right) & \text { if } z\left(y-y^{2}\right) \geq(x y+1)^{2}, \\
\frac{2}{y z}\left(\sqrt{z\left(y-y^{2}\right)}-1\right)^{2}+y-\frac{1}{z} & \text { if } z\left(y-y^{2}\right) \leq 1,\end{cases} \\
(x+1)\left(\frac{y}{x y+1}-\frac{1}{z}\right)-y+\frac{1}{z} & \text { if } z\left(y-y^{2}\right) \geq(x y+1)^{2},\end{cases}
\end{aligned}
$$

and the bounds (i) through (iii) follow from (5), (6), (8), and the last equation. 
Finally, in Case II we have

$$
\begin{aligned}
I_{2} & =\int_{0}^{z-1 / y}(u+1) d H_{n}^{a}(u) \int_{1 /(z-u)}^{y} \frac{d v}{(u v+1)^{2}} \\
& =\int_{0}^{z-1 / y}(u+1)\left(\frac{y}{u y+1}-\frac{1}{z}\right) d H_{n}^{a}(u) \\
& =\left.(u+1)\left(\frac{y}{u y+1}-\frac{1}{z}\right) H_{n}(u)\right|_{u=0} ^{u=z-1 / y}-\int_{0}^{z-1 / y} H_{n}^{a}(u)\left(\frac{y-y^{2}}{(u y+1)^{2}}-\frac{1}{z}\right) d u \\
& =-\int_{0}^{z-1 / y} H_{n}^{a}(u)\left(\frac{y-y^{2}}{(u y+1)^{2}}-\frac{1}{z}\right) d u .
\end{aligned}
$$

In the present case

$$
\begin{aligned}
& \int_{0}^{z-1 / y}\left|\frac{y-y^{2}}{(u y+1)^{2}}-\frac{1}{z}\right| d u \\
& = \begin{cases}\int_{0}^{z-1 / y}\left(-\frac{y-y^{2}}{(u y+1)^{2}}+\frac{1}{z}\right) d u & \text { if } z\left(y-y^{2}\right) \leq 1, \\
\int_{0}^{u_{0}}\left(\frac{y-y^{2}}{(u y+1)^{2}}-\frac{1}{z}\right) d u+\int_{u_{0}}^{z-1 / y}\left(\frac{1}{z}-\frac{y-y^{2}}{(u y+1)^{2}}\right) d u & \text { if } z\left(y-y^{2}\right) \geq 1,\end{cases} \\
& = \begin{cases}y-\frac{1}{z} & \text { if } z\left(y-y^{2}\right) \leq 1, \\
\frac{2}{y z}\left(\sqrt{z\left(y-y^{2}\right)}-1\right)^{2}+y-\frac{1}{z} & \text { if } z\left(y-y^{2}\right) \geq 1,\end{cases}
\end{aligned}
$$

and the bounds (iv) and (v) follow from (5), (7), (9), and the last equation.

The lower bound for (4) follows from the simple remark that

$$
\begin{aligned}
\sup _{x, y \in I, z \geq 1}\left|P_{n}^{a}(x, y, z)-L(x, y, z)\right| & \geq \sup _{x, y \in I}\left|P_{n}^{a}(x, y, \infty)-L(x, y, \infty)\right| \\
& =\sup _{x, y \in I}\left|\gamma_{a}\left(s_{n}^{a} \leq x, \tau^{n} \leq y\right)-\frac{\log (x y+1)}{\log 2}\right|,
\end{aligned}
$$

in conjunction with 3 . Hence, as stated, the optimal convergence rate $\mathcal{O}\left(g^{2 n}\right)$ to 0 as $n \rightarrow \infty$ for $\sup _{x, y \in I, z \geq 1}\left|P_{n}^{a}(x, y, z)-L(x, y, z)\right|$ follows.

Remark 1. The condition $z\left(y-y^{2}\right) \leq 1$ always holds when $z \leq 4, y \in I$. For $z>4$ it holds if and only if either

$$
y \leq \frac{1-\sqrt{1-4 / z}}{2}=\frac{2}{z(1+\sqrt{1-4 / z})}\left(\geq \frac{1}{z}\right)
$$

or

$$
y \geq \frac{1+\sqrt{1-4 / z}}{2}=\frac{2}{z(1-\sqrt{1-4 / z})}(\leq 1) .
$$


The condition $z\left(y-y^{2}\right) \geq 1$ does not hold when $z<4, y \in I$. For $z>4$ it holds if and only if

$$
\frac{2}{z(1+\sqrt{1-4 / z})} \leq y \leq \frac{2}{z(1-\sqrt{1-4 / z})} .
$$

The condition $z\left(y-y^{2}\right) \leq(x y+1)^{2}$ always holds when $z \leq 4,(x, y) \in I^{2}$. It also holds when $4<z \leq 8, z / 4-1 \leq x \leq 1, y \in I$. For $4<z \leq 8$ and $0 \leq x \leq z / 4-1$, or for $z>8$ and $x$ arbitrary in $I$, it holds if and only if either

$$
y \leq \frac{2}{z(1-2 x / z+\sqrt{1-4(x+1) / z})}\left(\geq \frac{2}{z(1+\sqrt{1-4 / z})}>\frac{1}{z-1}\right)
$$

or

$$
y \geq \frac{2}{z(1-2 x / z-\sqrt{1-4(x+1) / z})}\left(\leq \frac{2}{z(1-\sqrt{1-4 / z})}\right) .
$$

Finally, the condition $z\left(y-y^{2}\right) \geq(x y+1)^{2}$ does not hold when $z \leq 4,(x, y) \in I^{2}$, and when $4<z \leq 8, z / 4-1 \leq x \leq 1, y \in I$. For $4<z \leq 8,0 \leq x \leq z / 4-1$, or for $z>8$ and $x$ arbitrary in $I$, it holds if and only if

$$
\frac{2}{z(1-2 x / z-\sqrt{1-4(x+1) / z})} \leq y \leq \frac{2}{z(1-2 x / z+\sqrt{1-4(x+1) / z})} .
$$

\section{Two special cases}

Our main result allows us to derive the joint asymptotic distribution functions as $n \rightarrow \infty$ of the pairs $\left(\tau^{n}, u_{n+1}^{a}\right)$ and $\left(s_{n}^{a}, u_{n+1}^{a}\right)$.

\subsection{The pair $\left(\tau^{n}, u_{n+1}^{a}\right)$}

Letting $x=1$ we obtain the asymptotic distribution function as $n \rightarrow \infty$ of the pair $\left(\tau^{n}, u_{n+1}^{a}\right)$ under $\gamma_{a}, a \in I$. Denoting it by $M$, we have

$$
M(y, z)=L(1, y, z)= \begin{cases}\frac{1}{\log 2}\left(\log (y+1)-\frac{1}{z}\right) & \text { if } 1 /(z-1) \leq y \leq 1, \\ \frac{1}{\log 2}\left(\log y z+\frac{1}{y z}-1\right) & \text { if } 1 / z \leq y \leq 1 /(z-1), \\ 0 & \text { if } y \leq 1 / z\end{cases}
$$

for $(y, z) \in I \times[1, \infty)$. Clearly, the upper bounds (i) through (vi) in our theorem hold with obvious alterations, yielding again for any $a \in I$ a convergence rate $\mathcal{O}\left(g^{2 n}\right)$ as $n \rightarrow \infty$ for

$$
\sup _{y \in I, z \geq 1}\left|\gamma_{a}\left(\tau^{n} \leq y, u_{n+1}^{a} \leq z\right)-M(y, z)\right| .
$$


Since the distribution functions under $\gamma_{a}$ of both $\tau^{n}$ and $u_{n+1}^{a}$ converge as $n \rightarrow \infty$ to their limits at an optimal rate different from $\mathcal{O}\left(g^{2 n}\right)$, we should naturally ask ourselves whether a similar result also holds for their joint distribution function.

We will show that this is a correct guess indeed. In the present instance $(x=1)$, in Case I $(1 /(z-1) \leq y \leq 1)$ [cf. equation $(8)]$ we have

$$
\begin{aligned}
I_{2} & =\int_{0}^{1}(u+1)\left(\frac{y}{u y+1}-\frac{1}{z}\right) d H_{n}^{a}(u) \\
& =U^{n} f_{y, z}(a)-U^{\infty} f_{y, z}, \quad n \in \mathbb{N}, a \in I,
\end{aligned}
$$

with

$$
f_{y, z}(u)=(u+1)\left(\frac{y}{u y+1}-\frac{1}{z}\right), \quad u \in I .
$$

Here $U$ stands for the transition operator of all Markov chains $\left(s_{n}^{a}\right)_{n \in \mathbb{N}}, a \in I$, defined by

$$
U f(u)=\sum_{i \in \mathbb{N}_{+}} P_{i}(u) f\left(\frac{1}{x+i}\right), \quad u \in I,
$$

for any bounded complex-valued measurable function $f$ defined on $I$ while $U^{\infty} f=$ $\int_{I} f d \gamma$ (cf. [IK] pp. 65 and 136]). Further, in Case II $(1 / z \leq y \leq 1 /(z-1))$ we similarly have

$$
\begin{aligned}
I_{2} & =\int_{0}^{z-1 / y}(u+1)\left(\frac{y}{u y+1}-\frac{1}{z}\right) d H_{n}^{a}(u) \\
& =U^{n} g_{y, z}(a)-U^{\infty} g_{y, z}, \quad n \in \mathbb{N}, a \in I,
\end{aligned}
$$

with

$$
g_{y, z}(u)=I_{[0, z-1 / y]}(u)(u+1)\left(\frac{y}{u y+1}-\frac{1}{z}\right), \quad u \in I .
$$

As both $f_{y, z}$ and $g_{y, z}$ are Lipschitz functions on $I$, with maximal slopes uniformly bounded in the corresponding domains of variation of $(y, z)$, Theorem 2.2.8 in [IK] allows us to assert that in both Cases I and II for any $a \in I \backslash E$ we have

$$
\left|I_{2}\right|=\mathcal{O}\left(\lambda_{0}^{n}\right)
$$

as $n \rightarrow \infty$, with an absolute constant implied in $\mathcal{O}$, and this convergence rate to 0 of $I_{2}$ is optimal. By [5] we can then state the following result.

Proposition 1. For any $a \in I \backslash E$ we have

$$
\sup _{y \in I, z \geq 1}\left|\gamma_{a}\left(\tau^{n} \leq y, u_{n+1}^{a} \leq z\right)-M(y, z)\right|=\mathcal{O}\left(\lambda_{0}^{n}\right)
$$

as $n \rightarrow \infty$, with an absolute constant implied in $\mathcal{O}$ and optimal convergence rate. 
Remark 2. We might try to estimate the constant implied in $\mathcal{O}$ above as follows. Using Corollary 1.3 .11 in $[\mathrm{IK}]$ it is easy to check that

$$
\begin{aligned}
\gamma_{a}\left(\tau^{n} \leq y\right)-\frac{\log (y+1)}{\log 2} & =-(1-y) \int_{0}^{1} \frac{d H_{n}^{a}(s)}{s y+1} \\
& =y(1-y) \int_{0}^{1} \frac{H_{n}^{a}(s) d s}{(s y+1)^{2}}, \quad a, y \in I, n \in \mathbb{N} .
\end{aligned}
$$

Now, the result stated under (i) in Section 1 implies that

$$
\left|\gamma_{a}\left(\tau^{n} \leq y\right)-\frac{\log (y+1)}{\log 2}\right| \leq\left(\frac{\pi^{2}}{6}-\frac{1}{\log 2}\right) \lambda_{0}^{n-1} \min \left(y, \frac{1-y}{y+1}\right)
$$

for any $a, y \in I$ and $n \in \mathbb{N}_{+}$. It then follows that for any $a, y \in I$ and $n \in \mathbb{N}_{+}$we have

$$
\left|\int_{0}^{1} \frac{H_{n}^{a}(s) d s}{(s y+1)^{2}}\right| \leq \frac{2+\sqrt{2}}{2}\left(\frac{\pi^{2}}{6}-\frac{1}{\log 2}\right) \lambda_{0}^{n-1} .
$$

(We used the fact that $\min \left(y, \frac{1-y}{y+1}\right) \leq \frac{2+\sqrt{2}}{2} y(1-y), y \in I$.)

Turning now to equations (5) and (8) with $x=1$ (note that $H_{n}^{a}(1)=0, a \in I, n \in \mathbb{N}$ ), it is easy to see that in Case I we have

$$
\left|I_{2}\right| \leq\left(y-y^{2}+\frac{1}{z}\right)(2+\sqrt{2})\left(\frac{\pi^{2}}{6}-\frac{1}{\log 2}\right) \lambda_{0}^{n-1}
$$

for any $n \in \mathbb{N}_{+}$. The problem is that in Case II we cannot draw a similar conclusion since the integral in 9 is not over the whole interval $I$ ! Note that by the same Corollary 1.3.11 and Theorem 2.2.11 in [IK] we can only assert that

$$
\left|\int_{0}^{t} H_{n}^{a}(u) d u\right| \leq \kappa_{1} \lambda_{0}^{n-1}
$$

for any $a, t \in I$ and $n \in \mathbb{N}_{+}$.

\subsection{The pair $\left(s_{n}^{a}, u_{n+1}^{a}\right)$}

Letting $y=1$ we obtain the asymptotic distribution function as $n \rightarrow \infty$ of the pair $\left(s_{n}^{a}, u_{n+1}^{a}\right)$ under $\gamma_{a}, a \in I$. Denoting it by $N$, we have

$$
N(x, z)=L(x, 1, z)= \begin{cases}\frac{1}{\log 2}\left(\log (x+1)-\frac{x}{z}\right) & \text { if } z \geq x+1, \\ \frac{1}{\log 2}\left(\log z+\frac{1}{z}-1\right) & \text { if } 1 \leq z \leq x+1,\end{cases}
$$

for $(x, z) \in I \times[1, \infty)$. In the present case the upper bounds of

$$
\left|\gamma_{a}\left(s_{n}^{a} \leq x, u_{n+1}^{a} \leq z\right)-N(x, z)\right|
$$

corresponding to (i)-(iv) in our theorem reduce to 
(j) $\left(1-\frac{1}{z}\right) \sup _{0 \leq u \leq x}\left|H_{n}^{a}(u)\right|$ if $z \geq x+1$;

(jj) $\left(1-\frac{1}{z}\right) \sup _{0 \leq u \leq z-1}\left|H_{n}^{a}(u)\right|$ if $1 \leq z \leq x+1, x \in I$.

Clearly, since

$$
\begin{aligned}
\sup _{x \in I, z \geq 1}\left|\gamma_{a}\left(s_{n}^{a} \leq x, u_{n+1}^{a} \leq z\right)-N(x, z)\right| & \geq \sup _{x \in I}\left|\gamma_{a}\left(s_{n}^{a} \leq x\right)-N(x, \infty)\right| \\
& =\sup _{x \in I}\left|\gamma_{a}\left(s_{n}^{a} \leq x\right)-\frac{\log (x+1)}{\log 2}\right|,
\end{aligned}
$$

the optimal convergence rate to 0 as $n \rightarrow \infty$ of 11 is $\mathcal{O}\left(g^{2 n}\right)$ for any $a \in I$.

At the same time we can state the following result.

Proposition 2. Let $D=\left\{(x, z) \in \mathbb{R}^{2}: 1 \leq z \leq x+1, x \in I\right\}$. For any $a \in I \backslash E$ we have

$$
\sup _{(x, z) \in D}\left|\gamma_{a}\left(s_{n}^{a} \leq x, u_{n+1}^{a} \leq z\right)-N(x, z)\right|=\mathcal{O}\left(\lambda_{0}^{n}\right)
$$

as $n \rightarrow \infty$, with an absolute constant implied in $\mathcal{O}$ and optimal convergence rate.

For the proof it is sufficient to note that for $y=1$ equation 9 yields

$$
I_{2}=\int_{0}^{z-1}\left(1-\frac{u+1}{z}\right) d H_{n}^{a}(u),
$$

which by 10 is equal to $U^{n} g_{1, z}(a)-U^{\infty} g_{1, z}$ for any $n \in \mathbb{N}, a \in I$, and $(x, z) \in D$.

\section{References}

[IK] Iosifescu, M., Kraaikamp, C.: Metrical Theory of Continued Fractions. Kluwer, Dordrecht (2002) Zbl pre01926547 MR 1960327 